Beitrag des BDR

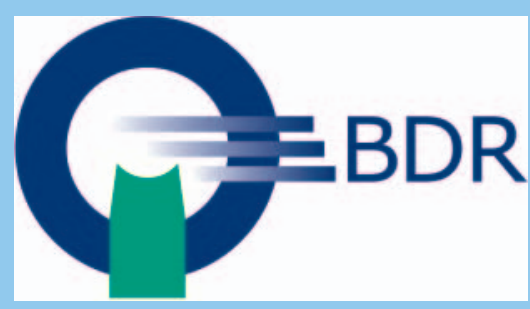

\title{
Neue Horizonte der Radiologie: Best Practice unter Pandemie-Bedingungen
}

In Berlin fand vom 17.-19. September das Symposium Neue Horizonte der Radiologie statt. Diese radiologische Fortbildungsveranstaltung wird alle zwei Jahre durchgeführt und in der Regel können etwa 250 Besucher und 60 Referenten begrüßt werden. Doch wie lässt sich das unter Pandemie-Bedingungen bewerkstelligen?

Viele Kongresse werden derzeit online durchgeführt. Die Referent*innen halten die Vorträge über diverse Streaming-Plattformen, Fragen der Teilnehmer*innen werden über den Chat gestellt. Die Vernetzung der Teilnehmer untereinander gibt es dadurch quasi nicht. Auch ein Gespräch mit den Experten beim Kaffee in der Pause ist in Online-Formaten nur schwer umzusetzen.

Deshalb hatten Professor Dr. Bernd Hamm und Professor Dr. Kay-Geert Hermann, die beiden wissenschaftlichen Leiter der Veranstaltung, gemeinsam mit dem EllingtonHotel ein Präsenzkonzept erarbeitet, dass mit allen Hygienebedingungen nach den Vorgaben des Berliner Senats konform ist. Aufgrund des sehr großen historischen Saals „Femina“ im Hotel konnte eine maximale Bestuhlung für 125 Personen bei 1,5 m Abstand zwischen allen Sitzen ermöglicht werden. Hinzu kamen Maskenpflicht in den Pausen, Verpflegung mit besonderen Hygienevorkehrungen, Desinfektionsspender in den öffentlichen Räumen sowie Handdesinfektion für alle Teilnehmer*innen. Das warme Wetter des Berliner Spätsommers tat sein Übriges und so konnte das Symposium auch durch die Unterstützung des Organisationsteams mit Frau Dr. Katharina Ziegeler und Frau Vera Schmidt an der Spitze mit Erfolg durchgeführt werden.

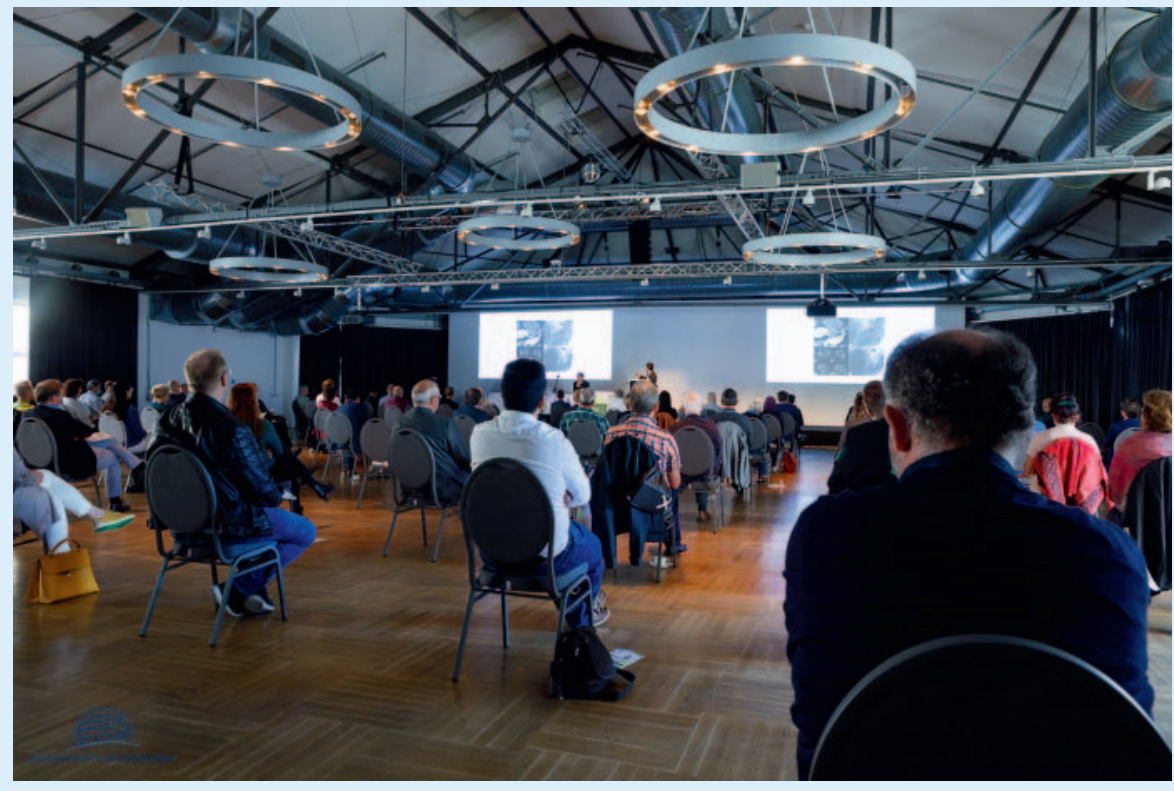

Mit ausreichendem Abstand beim Symposium NHdR.

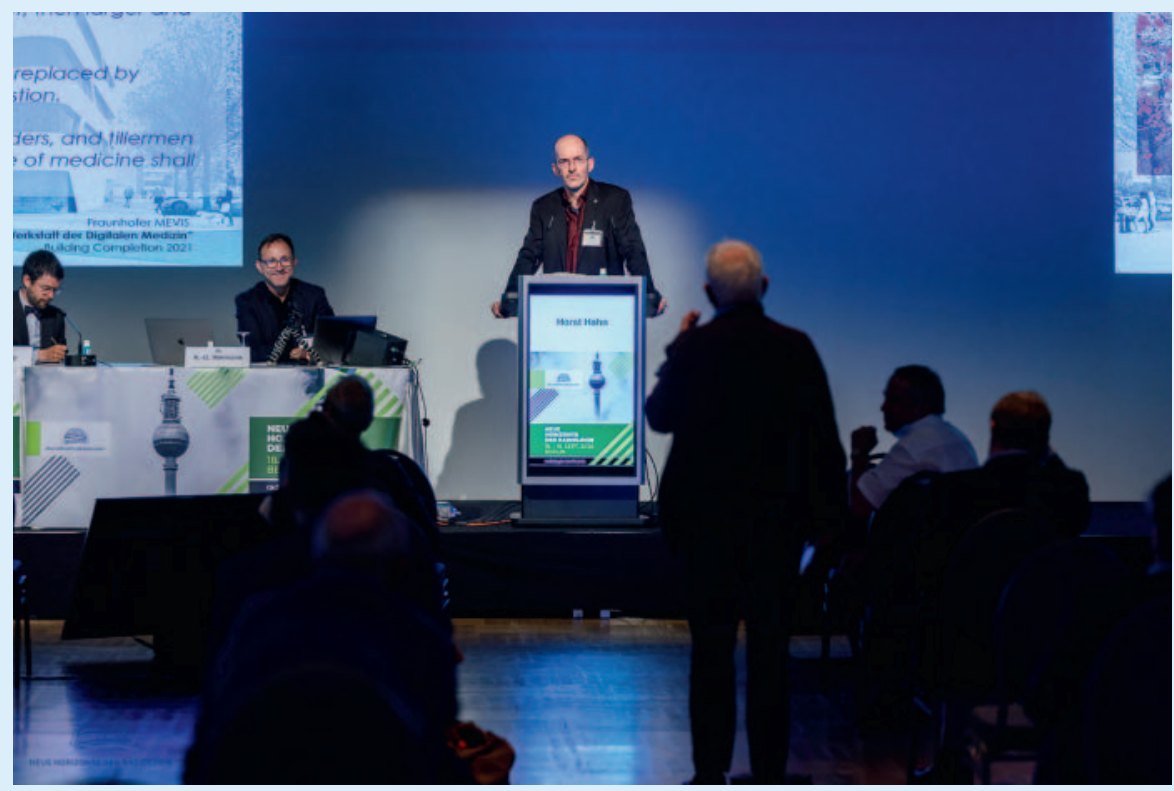

Diskussionen unter Pandemie-Bedingungen. 


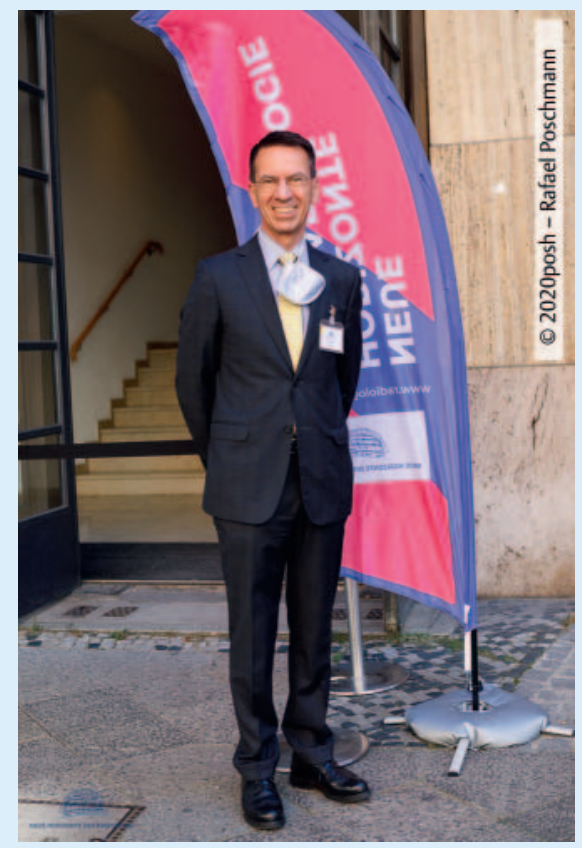

Themenschwerpunkte waren Anwendungen der künstlichen Intelligenz in der Radiologie sowie die urogenitale, abdominelle, kardiovaskuläre und muskuloskelettale Bildgebung. Des Weiteren gab es zwei Keynote-Lectures, einmal präsentiert von Herrn Professor Jörg Debatin zur Digitalen Medizin in Deutschland sowie von Frau Dr. Tonja Gaibler zum Update Radiologie und Recht.

Fester Bestandteil der Neuen Horizonte sind ebenfalls Workshops mit Hands-on Charakter. Die Themen Prostata-MRT, Rheuma-Bildgebung und Leber-MRT wurden angeboten und von den Teilnehmer*innen sehr gut angenommen.
Insgesamt war das Feedback überwältigend und durchweg positiv. Es sei ein sehr willkommenes Gefühl von „Normalität“ vermittelt worden nach diesem surrealen Jahr, sagten die Teilnehmer*innen und insbesondere die Referent*innen, die teilweise das erste Mal in 2020 auf einem Podium standen.

Retrospektiv gesehen hatten wir das besondere Glück, den richtigen Zeitpunkt zwischen den „Corona-Wellen“ für die Veranstaltung erwischt zu haben.

Prof. Dr. Bernd Hamm, Berlin Prof. Dr. Kay-Geert Hermann, Berlin 